\title{
Simple priorities and core stability in hedonic games*
}

\author{
Dinko Dimitrov ${ }^{\dagger}$ Peter Borm, Ruud Hendrickx \\ CentER and Department of Econometrics and Operations Research \\ Tilburg University, P.O. Box 90153, 5000 LE Tilburg, The Netherlands \\ Shao Chin Sung \\ School of Information Science \\ Japan Advanced Institute of Science and Technology \\ 1-1 Asahidai, Tatsunokuchi, Ishikawa 923-1292, Japan
}

January 2004

\begin{abstract}
In this paper we study hedonic games where each player views every other player either as a friend or as an enemy. Two simple priority criteria for comparison of coalitions are suggested, and the corresponding preference restrictions based on appreciation of friends and aversion to enemies are considered. It turns out that the first domain restriction guarantees non-emptiness of the strong core and the second domain restriction ensures non-emptiness of the weak core of the corresponding hedonic games. Moreover, an element of the strong core under friends appreciation can be found in polynomial time, while finding an element of the weak core under enemies aversion is NP-hard. We examine also the relationship between our domain
\end{abstract}

*The authors would like to thank Yongsheng Xu for helpful comments. Dinko Dimitrov gratefully acknowledges financial support from a Marie Curie Research Fellowship of the European Community programme "Improving the Human Research Potential and the Socio-Economic Knowledge Base" under contract number HPMF-CT-2002-02121.

†Corresponding author. E-mail address: d.a.dimitrov@uvt.nl 
restrictions and some sufficient conditions for non-emptiness of the core already known in the literature.

Journal of Economic Literature Classification Numbers: C71, A14, D20.

Keywords: additive separability, coalition formation, core stability, hedonic games, NP-completeness, priority.

\section{Introduction}

The study of the hedonic aspect of coalition formation goes back to Drèze and Greenberg (1980) who stress the dependence of a player's utility on the composition of members of her coalition. The formal model of a hedonic game was introduced by Banerjee, Konishi and Sönmez (2001) and Bogomolnaia and Jackson (2002). In their work, the focus on the identity of the members of a coalition determines the structure of the game: the latter consists of a preference ranking, for each player, over the coalitions to which that player belongs.

Despite the simplicity of the model, it turned out that the question of the existence of a core stable partition, that is, a partition of the set of all players for which there is no group of individuals who can all be better off by forming a new deviating coalition, does not have an easy answer. In this paper we restrict ourselves to hedonic games with separable preferences, i.e. games where the effect of a given player on another player's preferences is the same, regardless of which coalition the latter player is a member of. In such games every player partitions the society into desirable and undesirable coalitional partners (friends and enemies, respectively), and the division between friends and enemies guides the ordering of coalitions in the sense that adding a friend leads to a more preferable coalition, while adding an enemy leads to a less preferable coalition. 
As shown by Banerjee, Konishi and Sönmez (2001), non-emptiness of the core is not guaranteed even if one restricts separability to additive separability (players' preferences are representable by an additive separable utility function) and imposes in addition symmetry (i.e. the players have the same reciprocal values for each other). For an excellent study of the role of symmetric additive separable preferences for non-emptiness of the core of a hedonic game the reader is referred to Burani and Zwicker (2003).

In this paper we impose neither symmetry nor mutuality (i.e. the friendship among players is always mutual) on players' preferences. However, we restrict the domain of additive separable preferences by assuming that each player uses a simple priority criterion when comparing coalitions she may belong to. As a result, the class of additive separable preferences based on appreciation of friends and the class of additive separable preferences based on aversion to enemies are considered. The first preference domain corresponds to a situation in which every player in the game has very strong friends and very weak enemies: when comparing two coalitions she may belong to, a player who appreciates her friends pays attention first to the friends in either coalition. The coalition that contains more friends is declared by the player as better than the other, and if the two coalitions have the same number of friends, then the coalition with less enemies wins the comparison. The second preference domain displays a situation in which every player has very strong enemies and very weak friends, i.e. a player who is averse to her enemies looks first at the enemies in either coalition. The coalition that contains less enemies is declared by the player as better than the other, and if the two coalitions have the same number of enemies, then the number of friends is decisive for the comparison. Notice that both restrictions allow for indifferences in the corresponding rankings over coalitions. 
It turns out that friends appreciation is a sufficient condition for nonemptiness of the strong core and that enemies aversion guarantees nonemptiness of the weak core of the corresponding hedonic games. In addition, we show that an element of the strong core under friends appreciation can be found in polynomial time, while finding an element of the weak core under enemies aversion is NP-hard.

This paper serves three purposes. The first is providing positive results on the proposed preference domains: when the players are averse to their enemies a weak core stable coalition structure exists, and when the players appreciate their friends even the strong core is non-empty. The second is that our domain restrictions can be seen as specific extensions of the rudimentary information about one's opinion over the single players (viewed either as homogeneous enemies or as homogeneous friends) to an ordering over coalitions. Cechlárová and Romero-Medina (2001) propose a different way for extending preferences over single individuals to preferences over coalitions (that coincide with her preferences over the most attractive member in the corresponding coalitions) and show that the existence of a strong core stable partition of the players into coalitions is not guaranteed when indifferences are allowed. In contrast, we allow for indifferences and report a positive result on the preference domain based on appreciation of friends. Our third purpose is the analysis of the computational complexity of the problem for finding a core stable partition in hedonic games. For general hedonic games this problem is shown to be NP-hard; moreover, if one imposes anonymity (the players pay attention only to the size of the corresponding coalitions) the problem remains NP-hard even when only strict preferences are allowed (cf. Ballester (2003)). Cechlárová and Hajduková (2002) study the computational complexity for finding a strong core element for the specific extension 
of the preferences over individuals to preferences over coalitions proposed by Cechlárová and Romero-Medina (2001) and show that when ties are included the problem is NP-hard. Our domain restriction based on appreciation of friends allows for indifferences and it can be seen also as an attempt to conciliate additive separability with a weaker notion of anonymity (only the number of friends and the number of enemies count). We show that a strong core element can be found in polynomial time.

The outline of the paper is as follows. Section 2 introduces the formal model of a hedonic game and presents the formal definitions of our domains restrictions. We introduce the notions of an internally stable coalition and of a deviation stable collection of coalitions as our basic analytical tools in Section 3, and relate them to core stability. The set of internally stable coalitions has a special structure when restricting the preferences to friends appreciation or to enemies aversion. This special structure is studied in Section 4 and Section 5, respectively, where we present our core existence proofs as well. Section 6 is devoted to the computational complexity for finding a core stable element for hedonic games in our domains. In Section 7 we briefly comment on the relationship between the domain restriction based on appreciation of friends and the domain restrictions proposed by Cechlárová and Romero-Medina (2001) since the latter restrictions are also shown to give rise to strong core stable hedonic games. We consider also the relationship between the domain restriction based on aversion to enemies to other sufficient conditions shown to guarantee weak core stability of the corresponding games like the weak top coalition property of Banerjee, Konishi and Sönmez (2001) and the ordinal balancedness condition of Bogomolnaia and Jackson (2002). 


\section{Preliminaries}

Consider a finite set of players $N=\{1,2, \ldots, n\}$. A coalition is a non-empty subset of $N$. For each player $i \in N$, we denote by $\mathcal{N}_{i}=\{X \subseteq N \mid i \in X\}$ the collection of all coalitions containing $i$. A collection $\mathcal{C}$ of coalitions is called a coalition structure if $\mathcal{C}$ is a partition of $N$, i.e. the coalitions in $\mathcal{C}$ are pairwise disjoint and $\bigcup_{C \in \mathcal{C}} C=N$. By $\mathbf{C}^{N}$ we denote the set of all coalition structures of $N$. For each coalition structure $\mathcal{C} \in \mathbf{C}^{N}$ and each player $i \in N$, by $\mathcal{C}(i)$ we denote the coalition in $\mathcal{C}$ which contains $i$, i.e. $\{\mathcal{C}(i)\}=\mathcal{C} \cap \mathcal{N}_{i}$.

We assume that each player $i \in N$ is endowed with a preference $\succeq_{i}$ over $\mathcal{N}_{i}$, i.e. a binary relation over $\mathcal{N}_{i}$ which is reflexive, complete, and transitive. We denote by $P=\left(\succeq_{1}, \succeq_{2}, \ldots, \succeq_{n}\right)$ a profile of preferences $\succeq_{i}$ for all $i \in N$, and by $\mathcal{P}$ the set of all preference profiles. Moreover, we assume that the preference of each player $i \in N$ over coalition structures is purely hedonic, i.e. it is completely characterized by $\succeq_{i}$ in such a way that, for each $\mathcal{C}, \mathcal{C}^{\prime} \in \mathbf{C}^{N}$, player $i$ weakly prefers $\mathcal{C}$ to $\mathcal{C}^{\prime}$ if and only if $\mathcal{C}(i) \succeq_{i} \mathcal{C}^{\prime}(i)$.

A hedonic game is a pair $(N, P)$ consisting of a finite set $N$ of players and a preference profile $P \in \mathcal{P}$. This paper is devoted to the question whether there exists a coalition structure $\mathcal{C} \in \mathbf{C}^{N}$ which is stable in some sense. The corresponding stability notions are given in Section 3.

We now specify the preference domains that will be considered. For each $i \in N$, we let $G_{i}:=G\left(\succeq_{i}\right)=\left\{j \in N:\{i, j\} \succeq_{i}\{i\}\right\}$ be the set of friends of player $i$, and its complement $B_{i}=N \backslash G_{i}$ the set of enemies of player $i$. Notice that, from $\{i\} \succeq_{i}\{i\}$, we have $i \in G_{i}$ for each $i \in N$. The next definition suggests two ways of how each player $i$ ranks the sets in $\mathcal{N}_{i}$ depending on the numbers of her friends and enemies.

Definition 1 Let $P=\left(\succeq_{1}, \succeq_{2}, \ldots, \succeq_{n}\right) \in \mathcal{P}$ be a profile of players' prefer- 
ences.

- We say that $P$ is based on appreciation of friends if, for all $i \in N$ and all $X, Y \in \mathcal{N}_{i}$,

$$
X \succeq_{i} Y \Leftrightarrow\left\{\begin{array}{c}
\left|X \cap G_{i}\right|>\left|Y \cap G_{i}\right| \\
o r \\
\left|X \cap G_{i}\right|=\left|Y \cap G_{i}\right| \text { and }\left|X \cap B_{i}\right| \leq\left|Y \cap B_{i}\right| .
\end{array}\right.
$$

- We say that $P$ is based on aversion to enemies if, for all $i \in N$ and all $X, Y \in \mathcal{N}_{i}$,

$$
X \succeq_{i} Y \Leftrightarrow\left\{\begin{array}{c}
\left|X \cap B_{i}\right|<\left|Y \cap B_{i}\right| \\
\text { or } \\
\left|X \cap B_{i}\right|=\left|Y \cap B_{i}\right| \text { and }\left|X \cap G_{i}\right| \geq\left|Y \cap G_{i}\right| .
\end{array}\right.
$$

Thus, if the preference profile is based on appreciation of friends, we have a priority for friends when comparing two coalitions. If the preference profile is based on aversion to enemies, each player looks first at her enemies when comparing two coalitions. In the following, the set of all preference profiles based on appreciation of friends is denoted by $\mathcal{P}^{f}$, and the set of all preference profiles based on aversion to enemies is denoted by $\mathcal{P}^{e}$.

It is not difficult to see that if players' preferences are induced by either way suggested by Definition 1, then each player $i$ will be equipped with a preference relation over $\mathcal{N}_{i}$ with $G_{i}$ being its top and $B_{i} \cup\{i\}$ being its bottom. The next example illustrates this point.

Example 1 Let $N=\{1,2,3\}$ and $G_{1}=\{1,2\}, G_{2}=\{2\}, G_{3}=\{1,2,3\}$. Let $P=\left(\succeq_{1}, \succeq_{2}, \succeq_{3}\right) \in \mathcal{P}$. 
- If $P \in \mathcal{P}^{f}$, then

- the ranking over $\mathcal{N}_{1}$ is $\{1,2\} \succ_{1}\{1,2,3\} \succ_{1}\{1\} \succ_{1}\{1,3\}$,

- the ranking over $\mathcal{N}_{2}$ is $\{2\} \succ_{2}\{1,2\} \sim_{2}\{2,3\} \succ_{2}\{1,2,3\}$, and

- the ranking over $\mathcal{N}_{3}$ is $\{1,2,3\} \succ_{3}\{1,3\} \sim_{3}\{2,3\} \succ_{3}\{3\}$.

- If $P \in \mathcal{P}^{e}$, then

- the ranking over $\mathcal{N}_{1}$ is $\{1,2\} \succ_{1}\{1\} \succ_{1}\{1,2,3\} \succ_{1}\{1,3\}$,

- the ranking over $\mathcal{N}_{2}$ is $\{2\} \succ_{2}\{1,2\} \sim_{2}\{2,3\} \succ_{2}\{1,2,3\}$, and

- the ranking over $\mathcal{N}_{3}$ is $\{1,2,3\} \succ_{3}\{1,3\} \sim_{3}\{2,3\} \succ_{3}\{3\}$.

In fact, the preference profiles based on appreciation of friends and the preference profiles based on aversion to enemies belong to a more general class of preference profiles, namely the class of additive separable preferences.

Definition 2 A profile $P \in \mathcal{P}$ of players' preferences is additive separable if, for all $i \in N$, there exists a function $v_{i}: N \rightarrow \mathbb{R}$ such that for all $X, Y \in \mathcal{N}_{i}$,

$$
X \succeq_{i} Y \Leftrightarrow \sum_{j \in X} v_{i}(j) \geq \sum_{j \in Y} v_{i}(j)
$$

We denote the set of all additive separable preferences by $\mathcal{P}^{a s}$. For the preference profile $P \in \mathcal{P}^{f}$ in Example 1, one can take $v_{1}(1)=v_{1}(2)=$ $v_{2}(2)=v_{3}(1)=v_{3}(2)=v_{3}(3)=3$ and $v_{1}(3)=v_{2}(1)=v_{2}(3)=-1$. For the preference profile $P \in \mathcal{P}^{e}$ in the same example the choice can be $v_{1}(1)=$ $v_{1}(2)=v_{2}(2)=v_{3}(1)=v_{3}(2)=v_{3}(3)=1$ and $v_{1}(3)=v_{2}(1)=v_{2}(3)=-3$. More generally, when $P \in \mathcal{P}^{f}$, one can take, for each $i \in N, v_{i}(j)=n$ if $j \in G_{i}$, and $v_{i}(j)=-1$ otherwise; when $P \in \mathcal{P}^{e}$, one can take, for each $i \in N, v_{i}(j)=1$ if $j \in G_{i}$, and $v_{i}(j)=-n$ otherwise. Therefore, we have $\left(\mathcal{P}^{f} \cup \mathcal{P}^{e}\right) \subset \mathcal{P}^{a s}$.

All additive separable preference profiles are also separable. A profile $P \in \mathcal{P}$ of players' preferences is separable if, for every player $i \in N$ there is 
a partition $\left(G_{i}, B_{i}\right)$ of $N$ such that for every $j \in N$ and $X \in \mathcal{N}_{i}$ with $j \notin X$, we have $\left[X \cup\{j\} \succeq_{i} X \Leftrightarrow j \in G_{i}\right]$ and $\left[X \cup\{j\} \preceq_{i} X \Leftrightarrow j \in B_{i}\right]$. We denote the set of all separable preferences by $\mathcal{P}^{s}$. Hence, the relation among $\mathcal{P}^{f}, \mathcal{P}^{e}$, $\mathcal{P}^{a s}$, and $\mathcal{P}^{s}$ is as follows:

$$
\left(\mathcal{P}^{f} \cup \mathcal{P}^{e}\right) \subset \mathcal{P}^{a s} \subset \mathcal{P}^{s} \subset \mathcal{P}
$$

\section{Core stability and internal stability}

In this section, we introduce the concepts of weak and strong core stability and our main analytic tool, namely the concept of internal stability.

By $\mathbf{D}^{N}$ we denote the set of all collections of disjoint non-empty coalitions. For each $\mathcal{D} \in \mathbf{D}^{N}$ and for each $i \in \bigcup_{D \in \mathcal{D}} D$, we denote by $\mathcal{D}(i)$ the coalition in $\mathcal{D}$ containing $i$, i.e. $\{\mathcal{D}(i)\}=\mathcal{D} \cap \mathcal{N}_{i}$. Notice that the empty collection of coalitions belongs to $\mathbf{D}^{N}$, i.e. $\emptyset \in \mathbf{D}^{N}$. Observe further that each coalition structure is also a collection of non-empty disjoint coalitions, and thus, $\mathbf{C}^{N} \subseteq$ $\mathbf{D}^{N}$.

Definition 3 Let $P \in \mathcal{P}, X \subseteq N$ and $\mathcal{D} \in \mathbf{D}^{N}$.

- We say that $X$ is a strong deviation from $\mathcal{D}$ if $\emptyset \neq X \subseteq \bigcup_{D \in \mathcal{D}} D$, and $X \succ_{i} \mathcal{D}(i)$ for each $i \in X$.

- We say that $X$ is a weak deviation from $\mathcal{D}$ if $\emptyset \neq X \subseteq \bigcup_{D \in \mathcal{D}} D$, $X \succeq_{i} \mathcal{D}(i)$ for each $i \in X$, and $X \succ_{j} \mathcal{D}(j)$ for at least one $j \in X$.

By using these notions, we define now weak and strong core stability.

Definition 4 Let $P \in \mathcal{P}$ and $\mathcal{C} \in \mathbf{C}^{N}$.

- We say that $\mathcal{C}$ is weak core stable if a strong deviation from $\mathcal{C}$ does not exist. 
- We say that $\mathcal{C}$ is strong core stable if a weak deviation from $\mathcal{C}$ does not exist.

Notice that $\{X\} \in \mathbf{D}^{N}$ for every non-empty coalition $X \subseteq N$. Similar to the notion of core stability for coalition structures, we define below the notion of internal stability for coalitions.

Definition 5 Let $P \in \mathcal{P}$ and $X \subseteq N$ with $X \neq \emptyset$.

- We say that $X$ is weak internally stable if there is no $Y \subseteq X$ which is a strong deviation from $\{X\}$.

- We say that $X$ is strong internally stable if there is no $Y \subseteq X$ which is a weak deviation from $\{X\}$.

We denote by $W(N, P)$ the collection of all weak internally stable coalitions, and by $S(N, P)$ the collection of all strong internally stable coalitions. Observe that, for every $P \in \mathcal{P}$ and every $\mathcal{C} \in \mathbf{C}^{N}$, we have $\mathcal{C} \subseteq W(N, P)$ if $\mathcal{C}$ is weak core stable, and $\mathcal{C} \subseteq S(N, P)$ if $\mathcal{C}$ is strong core stable.

Recall that each $\succeq_{i}$ is reflexive, complete and transitive, i.e. the strict preference $\succ_{i}$ of each player $i \in N$ is also transitive. Having this in mind, the following lemma shows some kinds of transitivities in terms of deviations, which provides a connection between core stability and internal stability.

Lemma 1 Let $P \in \mathcal{P}, \mathcal{D} \in \mathbf{D}^{N}$, and $X, Y \subseteq N$.

- If $X$ is a strong deviation from $\mathcal{D}$, and if $Y$ is a strong deviation from $\{X\}$, then $Y$ is also a strong deviation from $\mathcal{D}$.

- If $X$ is a weak deviation from $\mathcal{D}$, and if $Y$ is a weak deviation from $\{X\}$, then $Y$ is also a weak deviation from $\mathcal{D}$. 
Proof. Suppose $X$ is a strong deviation from $\mathcal{D}$, and $Y$ is a strong deviation from $\{X\}$. Then, we have $\emptyset \neq Y \subseteq X \subseteq \bigcup_{D \in \mathcal{D}} D$. Since each $\succeq_{i}$ is reflexive, $Y$ is a proper subcoalition of $X$, i.e. $Y \subset X$. From $X \succ_{i} \mathcal{D}(i)$ and $Y \succ_{i} X$ for each $i \in Y$, we have $Y \succ_{i} \mathcal{D}(i)$ for each $i \in Y$. Therefore, $Y$ is a strong deviation from $\mathcal{D}$.

Suppose $X$ is a weak deviation from $\mathcal{D}$, and $Y$ is a weak deviation from $\{X\}$. Again, we have $\emptyset \neq Y \subseteq X \subseteq \bigcup_{D \in \mathcal{D}} D$ and $Y \subset X$. From $X \succeq_{i} \mathcal{D}(i)$ and $Y \succeq_{i} X$ for each $i \in Y$, we have $Y \succeq_{i} \mathcal{D}(i)$ for each $i \in Y$. Moreover, there exists a $j \in Y$ such that $Y \succ_{j} X$. By combining with $X \succeq_{j} \mathcal{D}(j)$, we have $Y \succ_{j} \mathcal{D}(j)$. Therefore, $Y$ is a weak deviation from $\mathcal{D}$.

Lemma 2 Let $P \in \mathcal{P}$ and $X \subseteq N$ with $X \neq \emptyset$.

- If $X \notin W(N, P)$, then there exists a strong deviation $Y$ from $\{X\}$ such that $Y \in W(N, P)$.

- If $X \notin S(N, P)$, then there exists a weak deviation $Y$ from $\{X\}$ such that $Y \in S(N, P)$.

Proof. Suppose $X \notin W(N, P)$ and every strong deviation $Y$ from $\{X\}$ is such that $Y \notin W(N, P)$. Let $Y$ be a strong deviation from $\{X\}$ with the smallest cardinality. Notice that $Y \neq \emptyset$, and by assumption, $Y \notin W(N, P)$. Thus, there exists a strong deviation $Z$ from $\{Y\}$. From Lemma $1, Z$ is also a strong deviation from $\{X\}$. However, since each $\succeq_{i}$ is reflexive, every strong deviation $Z$ from $\{Y\}$ must be a non-empty proper subcoalition of $Y$, i.e. $\emptyset \neq$ $Z \subset Y$. Thus, we have $0<|Z|<|Y|$, which contradicts the assumption that $Y$ is a strong deviation from $\{X\}$ with the smallest cardinality. Therefore, there exists a strong deviation $Y$ from $\{X\}$ such that $Y \in W(N, R)$ if $X \notin$ $W(N, P)$. By a similar argument, one can show that there exists a weak deviation $Y$ from $\{X\}$ such that $Y \in S(N, R)$ if $X \notin S(N, P)$. 
From Lemma 2, we obtain the following characterizations of core stability in terms of internal stability.

Theorem 1 Let $P \in \mathcal{P}$. For every $\mathcal{C} \in \mathbf{C}^{N}$,

- $\mathcal{C}$ is weak core stable if and only if there does not exist any strong deviation from $\mathcal{C}$ which is weak internally stable,

- $\mathcal{C}$ is strong core stable if and only if there does not exist any weak deviation from $\mathcal{C}$ which is strong internally stable.

This is a very useful theorem because, when weak (strong) core stability is under consideration, the theorem allows us to concentrate only on strong (weak) deviations which are weak (strong) internally stable.

In Sections 4 and 5, we show that a strong core stable coalition structure exists for each $P \in \mathcal{P}^{f}$, and a weak core stable coalition structure exists for each $P \in \mathcal{P}^{e}$. By summing up the above arguments, we come to the following concepts, which allow us to provide constructive existence proofs.

Definition 6 Let $P \in \mathcal{P}$ and $\mathcal{D} \in \mathbf{D}^{N}$.

- We say that $\mathcal{D}$ is weak deviation stable if, for each $\mathcal{C} \in \mathbf{C}^{N}$ with $\mathcal{D} \subseteq \mathcal{C}$, there does not exist any strong deviation $X$ from $\mathcal{C}$ such that $X \in W(N, P)$ and $X \cap\left(\bigcup_{D \in \mathcal{D}} D\right) \neq \emptyset$.

- We say that $\mathcal{D}$ is strong deviation stable if, for each $\mathcal{C} \in \mathbf{C}^{N}$ with $\mathcal{D} \subseteq \mathcal{C}$, there does not exist any weak deviation $X$ from $\mathcal{C}$ such that $X \in S(N, P)$ and $X \cap\left(\bigcup_{D \in \mathcal{D}} D\right) \neq \emptyset$.

Observe that, by definition, for each $\mathcal{D} \in \mathbf{D}^{N}, \mathcal{D} \subseteq W(N, P)$ if $\mathcal{D}$ is weak deviation stable, and $\mathcal{D} \subseteq S(N, P)$ if $\mathcal{D}$ is strong deviation stable. Moreover, 
from Theorem 1, the following corollary can be obtained immediately.

Corollary 1 Let $P \in \mathcal{P}$. For every $\mathcal{C} \in \mathbf{C}^{N}$,

- $\mathcal{C}$ is weak core stable if and only if $\mathcal{C}$ is weak deviation stable, and

- $\mathcal{C}$ is strong core stable if and only if $\mathcal{C}$ is strong deviation stable.

A sketch of our constructions of core stable coalition structures in the following sections looks as follows:

- start with the empty collection of coalitions, which is weak (and also strong) deviation stable;

- construct a new weak (strong) deviation stable collection of disjoint non-empty coalitions by including a weak (strong) internally stable coalition, and repeat this until a coalition structure (a partition of $N$ ) is obtained.

So that, from Corollary 1, we will finally obtain a weak (strong) core stable coalition structure.

\section{Appreciation of friends}

In this section, we show the strong core stability of all hedonic games with preference profiles belonging to $\mathcal{P}^{f}$.

Let us first show a characterization of strong internally stable coalitions (Lemma 3) and the structure of the collection $S(N, P)$ of all strong internally stable coalitions (Lemma 4). 
Let $X \subseteq N$ with $X \neq \emptyset$, and $i \in X$. For each positive integer $t$, let $G_{i}^{t}(X) \subseteq X$ be a set defined as follows:

$$
\begin{aligned}
G_{i}^{1}(X) & =G_{i} \cap X \\
G_{i}^{t+1}(X) & =\bigcup_{j \in G_{i}^{t}(X)}\left(G_{j} \cap X\right) .
\end{aligned}
$$

Let $G_{i}^{*}(X)=G_{i}^{t}(X)$ for a positive integer $t$ such that $G_{i}^{t}(X)=G_{i}^{t+1}(X)$. Notice that $G_{i}^{n}(X)=G_{i}^{n+1}(X)$ for each $X \subseteq N$ and each $i \in X$. Also notice that $G_{i}^{*}(X) \subseteq X$, and $G_{i}^{*}(Y) \subseteq G_{i}^{*}(X)$ for each $Y \subseteq X$.

The following lemma provides a characterization of strong internally stable coalitions.

Lemma 3 Let $P \in \mathcal{P}^{f}$ and $X \subseteq N$ with $X \neq \emptyset$. Then, $X \in S(N, P)$ if and only if $G_{i}^{*}(X)=X$ for each $i \in X$.

Proof. Suppose $X \in S(N, P)$ and there exists $i \in X$ such that $G_{i}^{*}(X) \neq X$. Since $G_{i}^{*}(X) \subseteq X$ for each $i \in X$, we have $X \backslash G_{i}^{*}(X) \neq \emptyset$. Because $\left|X \cap G_{j}\right|=\left|G_{i}^{*}(X) \cap G_{j}\right|$ for each $j \in G_{i}^{*}(X)$ and since $X \backslash G_{i}^{*}(X)$ is nonempty, we have $\left|X \cap B_{j}\right|>\left|G_{i}^{*}(X) \cap B_{j}\right|$ for each $j \in G_{i}^{*}(X)$. Therefore, from $P \in \mathcal{P}^{f}$, we have $G_{i}^{*}(X) \succ_{j} X$ for each $j \in G_{i}^{*}(X)$. That is, $G_{i}^{*}(X)$ is a strong deviation from $\{X\}$, and is also a weak deviation from $\{X\}$. This contradicts the assumption that $X \in S(N, P)$.

Suppose $X \subseteq N$ with $X \neq \emptyset$ and is such that $G_{i}^{*}(X)=X$ for each $i \in X$. Let $Y \subset X$ be a non-empty proper subcoalition of $X$. Then, we have $G_{i}^{*}(X) \backslash Y \neq \emptyset$ for each $i \in Y$, and thus, there exists a $j \in Y$ such that $G_{j} \cap(X \backslash Y) \neq \emptyset$. That is, $\left|X \cap G_{j}\right|>\left|Y \cap G_{j}\right|$, and from $P \in \mathcal{P}^{f}$, we have $X \succ_{j} Y$ for some $j \in Y$. It follows that there is no subcoalition of $X$ which is a weak deviation from $\{X\}$. Therefore, we have $X \in S(N, P)$. 
Next, we show a useful property of the set of all strong internally stable coalitions.

Lemma 4 Let $P \in \mathcal{P}^{f}$. If $X, Y \in S(N, P)$ with $X \cap Y \neq \emptyset$, then $X \cup Y \in$ $S(N, P)$.

Proof. Suppose $X, Y \in S(N, P)$ with $X \cap Y \neq \emptyset$. From Lemma 3, it suffices to show that $G_{i}^{*}(X \cup Y)=X \cup Y$ for each $i \in X \cup Y$. Recall that we have, for each non-empty $Z \subseteq N$ and each $i \in Z, G_{i}^{*}(Z) \subseteq Z$, and $G_{i}^{*}(Z) \subseteq G_{i}^{*}\left(Z^{\prime}\right)$ if $Z \subseteq Z^{\prime}$. Thus $G_{i}^{*}(X) \cup G_{i}^{*}(Y) \subseteq G_{i}^{*}(X \cup Y) \subseteq X \cup Y$ for each $i \in X \cup Y$. In the following, we show that $X \cup Y \subseteq G_{i}^{*}(X \cup Y)$ for each $i \in X \cup Y$.

Let $i \in X \cap Y$. By assumption, $G_{i}^{*}(X)=X$ and $G_{i}^{*}(Y)=Y$, and thus, we have $X \cup Y=G_{i}^{*}(X) \cup G_{i}^{*}(Y) \subseteq G_{i}^{*}(X \cup Y)$ for each $i \in X \cap Y$.

Let $i \in X \backslash Y$. By assumption, $G_{i}^{*}(X)=X$, and thus, $X \subseteq G_{i}^{*}(X \cup Y)$. Let $j \in X \cap Y$. Notice that such a $j$ exists by assumption. Then $j \in$ $G_{i}^{*}(X \cup Y)$, and by definition $G_{j}^{*}(X \cup Y) \subseteq G_{i}^{*}(X \cup Y)$. Since $Y=G_{j}^{*}(Y) \subseteq$ $G_{j}^{*}(X \cup Y)$, we have $Y \subseteq G_{i}^{*}(X \cup Y)$. Thus, $X \cup Y \subseteq G_{i}^{*}(X \cup Y)$ for each $i \in X \backslash Y$. By the same argument, one can show that $X \cup Y \subseteq G_{i}^{*}(X \cup Y)$ for each $i \in Y \backslash X$.

Now we can conclude that $X \cup Y \in S(N, P)$ if $X, Y \in S(N, P)$ with $X \cap Y \neq \emptyset$, and the proof is completed.

Having described, for games with preference profiles belonging to $\mathcal{P}^{f}$, a characterization of strong internally stable coalitions and the structure of the collection $S(N, P)$, we redirect our attention to the problem of core stability of such games.

For each $M \subseteq N$, we denote by $S^{M}(N, P)$ the collection of all strong internally stable coalitions which are subsets of $M$, i.e. $S^{M}(N, P)=\{X \in$ $S(N, P) \mid X \subseteq M\}$. Let $G S^{M}(N, P)$ be the collection of all largest coalitions among coalitions belonging to $S^{M}(N, P)$, i.e. $G S^{M}(N, P)=\{X \in$ 
$S^{M}(N, P)|| X|\geq| Y \mid$ for all $\left.Y \in S^{M}(N, P)\right\}$. Obviously, $S^{N}(N, P)=$ $S(N, P)$. Notice that $\{i\} \in S^{M}(N, P)$ for each $i \in M$. Hence, $S^{M}(N, P)$ and $G S^{M}(N, P)$ are non-empty whenever $M$ is non-empty.

The following proposition suggests a way for extending a strong deviation stable collection of disjoint coalitions for $P \in \mathcal{P}^{f}$.

Proposition 1 Let $P \in \mathcal{P}^{f}, \mathcal{D} \in \mathbf{D}^{N} \backslash \mathbf{C}^{N}$, and $M=N \backslash\left(\bigcup_{D \in \mathcal{D}} D\right)$. If $\mathcal{D}$ is strong deviation stable, then $\mathcal{D} \cup\left\{D^{\prime}\right\}$ is strong deviation stable for each $D^{\prime} \in G S^{M}(N, P)$.

Proof. Suppose $\mathcal{D}$ is strong deviation stable and let $D^{\prime} \in G S^{M}(N, P)$. Observe that $\mathcal{D} \cup\left\{D^{\prime}\right\} \in \mathbf{D}^{N}$ and $D^{\prime} \in G S^{M}(N, P) \subseteq S^{M}(N, P) \subseteq S(N, P)$. In the following we show that $\mathcal{D} \cup\left\{D^{\prime}\right\}$ is strong deviation stable.

Since $\mathcal{D}$ is strong deviation stable, every $X \in S(N, P)$ with $X \cap \bigcup_{D \in \mathcal{D}} D \neq$ $\emptyset$ cannot be a weak deviation from any $\mathcal{C} \in \mathbf{C}^{N}$ such that $\left(\mathcal{D} \cup\left\{D^{\prime}\right\}\right) \subseteq \mathcal{C}$. Thus, a strong internally stable coalition $X$ is a weak deviation from some $\mathcal{C} \in \mathbf{C}^{N}$ such that $\left(\mathcal{D} \cup\left\{D^{\prime}\right\}\right) \subseteq \mathcal{C}$ only if $X \subseteq M$. Let $X \in S(N, P)$ with $X \subseteq M$ and $X \cap D^{\prime} \neq \emptyset$. From $D^{\prime} \in S(N, P)$ and Lemma 4, we have $X \cup D^{\prime} \in S(N, P)$. Since $X, D^{\prime} \subseteq M$, we have $X \cup D^{\prime} \in S^{M}(N, P)$. Moreover, if $X \subseteq D^{\prime}$, then, from $D^{\prime} \in S(N, P), X$ cannot be a weak deviation from $\left\{D^{\prime}\right\}$, and thus $X$ cannot be a weak deviation from any $\mathcal{C} \in \mathbf{C}^{N}$ such that $\left(\mathcal{D} \cup\left\{D^{\prime}\right\}\right) \subseteq \mathcal{C}$. Then, if $X \nsubseteq D^{\prime}$ we have $\left|X \cup D^{\prime}\right|>\left|D^{\prime}\right|$, which contradicts $D^{\prime} \in G S^{M}(N, P)$. Therefore, $\mathcal{D} \cup\left\{D^{\prime}\right\}$ is strong deviation stable.

Our main result in this section is the following.

Theorem 2 For each $P \in \mathcal{P}^{f}$, a strong core stable coalition structure exists. Proof. Extend the empty collection of coalitions (which is strong deviation stable) to a strong deviation stable coalition structure of $N$ in the way suggested by Proposition 1. Then, from Corollary 1, such a coalition structure 
is strong core stable.

\section{$5 \quad$ Aversion to enemies}

The way of proving the existence of a core stable partition for the case of appreciation of friends can be used also for the case of aversion to enemies.

First, we show a characterization of weak internally stable coalitions when $P \in \mathcal{P}^{e}$.

Lemma 5 Let $P \in \mathcal{P}^{e}$ and $X \subseteq N$ with $X \neq \emptyset$. Then, $X \in W(N, P)$ if and only if $G_{i}^{1}(X)=X$ for each $i \in X$.

Proof. If $X \subseteq N$ with $X \cap B_{i} \neq \emptyset$ for some $i \in X$, then, from $\{i\} \succ_{i} X,\{i\}$ is a strong deviation from $\{X\}$, i.e. $X \notin W(N, P)$. Thus, $X \subseteq G_{i}$ for each $i \in X$ if $X \in W(N, P)$. Obviously, $G_{i}^{1}(X)=X$ if $X \subseteq G_{i}$.

Suppose $X \subseteq N$ with $X \neq \emptyset$ is such that $G_{i}^{1}(X)=X$ for each $i \in X$. Let $Y \subset X$ be a non-empty proper subcoalition of $X$. Then, we have $\left|X \cap B_{i}\right|=$ $\left|Y \cap B_{i}\right|=0$ and $\left|X \cap G_{i}\right|>\left|Y \cap G_{i}\right|$ for each $i \in Y$, i.e. $X \succ_{i} Y$. Thus, there is no subcoalition of $X$ is a strong deviation from $\{X\}$, i.e. $X \in W(N, P)$.

In other words, $W(N, P)$ is the collection of all non-empty coalitions of players in $N$ such that, for each of these coalitions, the players like each other under preference profile $P \in \mathcal{P}^{e}$.

As analogy to $S^{M}(N, P)$ and $G S^{M}(N, P)$, we define $W^{M}(N, P)=\{X \in$ $W(N, P) \mid X \subseteq M\}$ and $G W^{M}(N, P)=\left\{X \in W^{M}(N, P)|| X|\geq| Y \mid\right.$ for all $\left.Y \in W^{M}(N, P)\right\}$ for each $M \subseteq N$. Again, $W^{M}(N, P)$ and $G W^{M}(N, P)$ are non-empty whenever $M$ is non-empty. 
The following proposition suggests a way for extending a weak deviation stable collection of disjoint coalitions for $P \in \mathcal{P}^{e}$.

Proposition 2 Let $P \in \mathcal{P}^{e}, \mathcal{D} \in \mathbf{D}^{N} \backslash \mathbf{C}^{N}$, and $M=N \backslash\left(\bigcup_{D \in \mathcal{D}} D\right)$. Then, if $\mathcal{D}$ is weak deviation stable, then $\mathcal{D} \cup\left\{D^{\prime}\right\}$ is weak deviation stable for each $D^{\prime} \in G W^{M}(N, P)$.

Proof. The proof is similar to the proof of Proposition 1. Again, observe that $\mathcal{D} \cup\left\{D^{\prime}\right\} \in \mathbf{D}^{N}$ and $D^{\prime} \in G W^{M}(N, P) \subseteq W^{M}(N, P) \subseteq W(N, P)$. In the following we show that $\mathcal{D} \cup\left\{D^{\prime}\right\}$ is weak deviation stable.

Since $\mathcal{D}$ is weak deviation stable, every $X \in W(N, P)$ with $X \cap \bigcup_{D \in \mathcal{D}} D \neq$ $\emptyset$ cannot be a strong deviation from any $\mathcal{C} \in \mathbf{C}^{N}$ such that $\left(\mathcal{D} \cup\left\{D^{\prime}\right\}\right) \subseteq \mathcal{C}$. Thus, a weak internally stable coalition $X$ is a strong deviation from some $\mathcal{C} \in \mathbf{C}^{N}$ such that $\left(\mathcal{D} \cup\left\{D^{\prime}\right\}\right) \subseteq \mathcal{C}$ only if $X \subseteq M$. Let $X \in S(N, P)$ with $X \subseteq M$ and $X \cap D^{\prime} \neq \emptyset$. From Lemma 5, we have $\left|X \cap B_{i}\right|=\left|D^{\prime} \cap B_{i}\right|=0$ for each $i \in X \cap D^{\prime}$. Then, if $|X| \leq\left|D^{\prime}\right|$, then $D^{\prime} \succeq_{i} X$ for each $i \in$ $X \cap D^{\prime}$, and thus, $X$ cannot be a strong deviation for any $\mathcal{C} \in \mathbf{C}^{N}$ with $\mathcal{D} \cup\left\{D^{\prime}\right\} \subseteq C$. Moreover, from $D^{\prime} \in G W^{M}(N, P)$, we have $|X| \leq\left|D^{\prime}\right|$ for every $X \in W^{M}(N, P)$. Therefore, $\mathcal{D} \cup\left\{D^{\prime}\right\}$ is weak deviation stable.

With the help of this proposition, we are able to present our main result in this section.

Theorem 3 For each $P \in \mathcal{P}^{e}$, a weak core stable coalition structure exists. Proof. Extend the empty collection of coalitions (which is weak deviation stable) to a weak deviation stable coalition structure of $N$ in the way suggested by Proposition 2. Then, from Corollary 1, such a coalition structure is weak core stable.

The next example shows that the strong core may be empty when the players are averse to their enemies. 
Example 2 Consider $N=\{1,2,3\}$ under aversion to enemies with $G_{1}=$ $\{1,2\}, G_{2}=\{1,2,3\}$, and $G_{3}=\{2,3\}$. Clearly, the candidates for strong stable coalition structures should be weak stable as well. As it can be easily seen, the game has two weak core stable elements: $\{\{1,2\},\{3\}\}$ and $\{\{1\},\{2,3\}\}$. Notice that $\{2,3\}$ and $\{1,2\}$ are weak stable deviations from $\{\{1,2\},\{3\}\}$ and $\{\{1\},\{2,3\}\}$, respectively. Therefore, no strong core stable coalition structure exists.

\section{Computational complexity}

In this section, the computational complexity for finding core stable coalition structures is considered.

We start with a lemma for the case of aversion to enemies.

Lemma 6 For every $P \in \mathcal{P}^{e}$ and every $\mathcal{C} \in \mathbf{C}^{N}, G W(N, P) \cap \mathcal{C} \neq \emptyset$ if $\mathcal{C}$ is weak core stable.

Proof. Let $\mathcal{C} \in \mathbf{C}^{N}$ be such that $G W(N, P) \cap \mathcal{C}=\emptyset$, and let $X \in G W(N, P)$. Then, we have $|X|>|\mathcal{C}(i)|$ for each $i \in X$. From Lemma $5, X \subseteq G_{i}$ for each $i \in X$, i.e. $\left|X \cap B_{i}\right|=0$ for each $i \in X$. Thus, $X \succ_{i} \mathcal{C}(i)$ for each $i \in X$, i.e. $X$ is a strong deviation from $\mathcal{C}$. Therefore, $\mathcal{C}$ is not weak core stable.

This lemma allows us to formulate our result on computational complexity for finding any weak core stable partition in hedonic games with enemy averse preferences.

Theorem 4 When $P \in \mathcal{P}^{e}$, the problem of finding a weak core stable coalition structure is NP-hard.

Proof. The NP-hardness is shown by reduction from the Maximum Clique Problem, which is know to be NP-hard. 
Maximum Clique Problem: For a given undirected graph $H=(V, E)$, find a clique with the maximum cardinality in $H$, where a clique $K$ in $H$ is a subset of $V$ such that $\{i, j\} \in E$ for every $i, j \in K$ with $i \neq j$.

Let $H$ be an undirected graph. Without loss of generality, we assume that $V=\{1,2, \ldots, n\}$. Consider the hedonic game $(N, P)$ with $P \in \mathcal{P}^{e}$ such that $N=\{1,2, \ldots, n\}$ and $G_{i}=\{i\} \cup\{j \in N \mid\{i, j\} \in E\}$ for each $i \in N$. Notice that, from Lemma 5 , for each $X \subseteq N, X \in W(N, P)$ if and only if $X$ is a clique in $H$, and $G W(N, P)$ is the set of all cliques with the maximum cardinality. Then, from Lemma 6 , to each weak core stable coalition structure belongs at least one clique $X$ with the maximum cardinality. This implies that the problem of finding weak core stable coalition structure when $P \in \mathcal{P}^{e}$ is at least as difficult as finding a clique with the maximum cardinality in an undirected graph. Therefore, the problem of finding a weak core stable coalition structure when $P \in \mathcal{P}^{e}$ is NP-hard.

Remark 1 Notice that, from the proof of Theorem 4, the problem of finding a weak core stable coalition structure when $P \in P^{e}$ remains NP-hard even if the mutuality condition " $j \in G_{i}$ if and only if $i \in G_{j}$ " is imposed.

In the following, we show that when $P \in \mathcal{P}^{f}$, a strong core stable coalition structure can be found in polynomial time, by taking a graph theoretical approach.

Theorem 5 When $P \in \mathcal{P}^{f}$, a strong core stable coalition structure can be found in polynomial time.

Proof. Let $N=\{1,2, \ldots, n\}, P \in \mathcal{P}^{f}$, and let $H_{(N, P)}=(V, E)$ be a directed graph such that $V=N$ and $E=\left\{\{i, j\} \in N \times N \mid i \neq j, j \in G_{i}\right\}$. Observe that, from the definition of $G_{i}^{*}$ (in Section 4), for every $X \subseteq N$ and $i, j \in X$, we have $j \in G_{i}^{*}(X)$ if and only if there exists a sequence 
$k_{1}, k_{2}, \ldots, k_{m}$ for some $m \geq 1$ such that $k_{1}, k_{2}, \ldots, k_{m} \in X$ and $k_{\ell+1} \in G_{k_{\ell}}$ for each $1 \leq \ell \leq m-1$. Thus, $j \in G_{i}^{*}(X)$ if and only if there exists a directed path from $i$ to $j$ via vertices belonging to $X$. From Lemma 3, we have $X \in S(N, P)$ if and only if, for each $i, j \in X$ with $i \neq j$, there exist directed paths via vertices belonging to $X$ from $i$ to $j$ and from $j$ to $i$. That is, $X \in S(N, P)$ if and only if the induced subgraph of $H_{(N, P)}$ by $X$ is strongly connected, where the induced subgraph $H_{X}$ of $H_{(N, P)}$ by $X$ is the directed graph such that $V_{X}=X$ and $E_{X}=E \cap(X \times X)$. Moreover, $X \in G S(N, P)$ if and only if the induced subgraph of $H_{(N, P)}$ by $X$ is a strongly connected component in $H_{(N, P)}$ with the largest number of vertices. From Theorem 2, a strong core stable coalition structure can be found by repeating the following algorithm:

- Set $M:=N$ and $\mathcal{C}:=\emptyset$.

- Repeat the following until $M=\emptyset$ :

- Find a set $X \subseteq M$ such that the induced subgraph of $H_{(M, P)}$ by $X$ is a strongly connected component in $H_{(M, P)}$ with the largest number of vertices.

- Set $M:=M \backslash X$ and $\mathcal{C}:=\mathcal{C} \cup\{X\}$.

- Return $\mathcal{C}$.

Notice that the "Repeat" loop runs at most $n$ times. Moreover, an algorithm for finding all strongly connected components of a directed graph (i.e. a strong decomposition of a directed graph) is proposed by Tarjan (1972), which has running time $O\left(n^{2}\right)$. Therefore, a strong core stable coalition structure can be found in $O\left(n^{3}\right)$ time. 


\section{Comparisons}

Let us now consider the relationship between our domain restrictions and some other properties that have been shown to guarantee existence of core stable coalition structures.

We start with the strong core stability for the case of appreciation of friends. Up till now, strong core stability of hedonic games has been shown to exist only on the two preference domains proposed by Cechlárová and Romero-Medina (2001). These authors study hedonic games in which the ranking over coalitions for each player is guided either by her most preferred member of the group or by her least preferred member of the group. However, these authors show strong core existence only for the case when the preferences of the players over single members are strict, which, in turn, excludes the possibility of indifferences when coalitions are compared. Moreover, Cechlárová and Romero-Medina (2001) provide an example of a game with an empty strong core when ties are incorporated in the preference domain. In contrast, we do allow for indifferences and show strong core existence.

Consider now the weak core stability for the case of aversion to enemies. We first construct an example of a hedonic game with such kind of preferences. After that we introduce two sufficient conditions for non-emptiness of the weak core already known in the literature (the weak top coalition property of Banerjee, Konishi and Sönmez (2001) and the ordinal balancedness condition of Bogomolnaia and Jackson (2002)) and show that the constructed hedonic game satisfies none of them.

Every player in the next example is indifferent among coalitions on the same row and, for each $i \in N$, the top row corresponds to $G_{i}$ and the bottom 
row corresponds to $B_{i} \cup\{i\}$.

Example 3 Let $N=\{1,2,3,4,5\}$ and the preferences of the players be as follows:

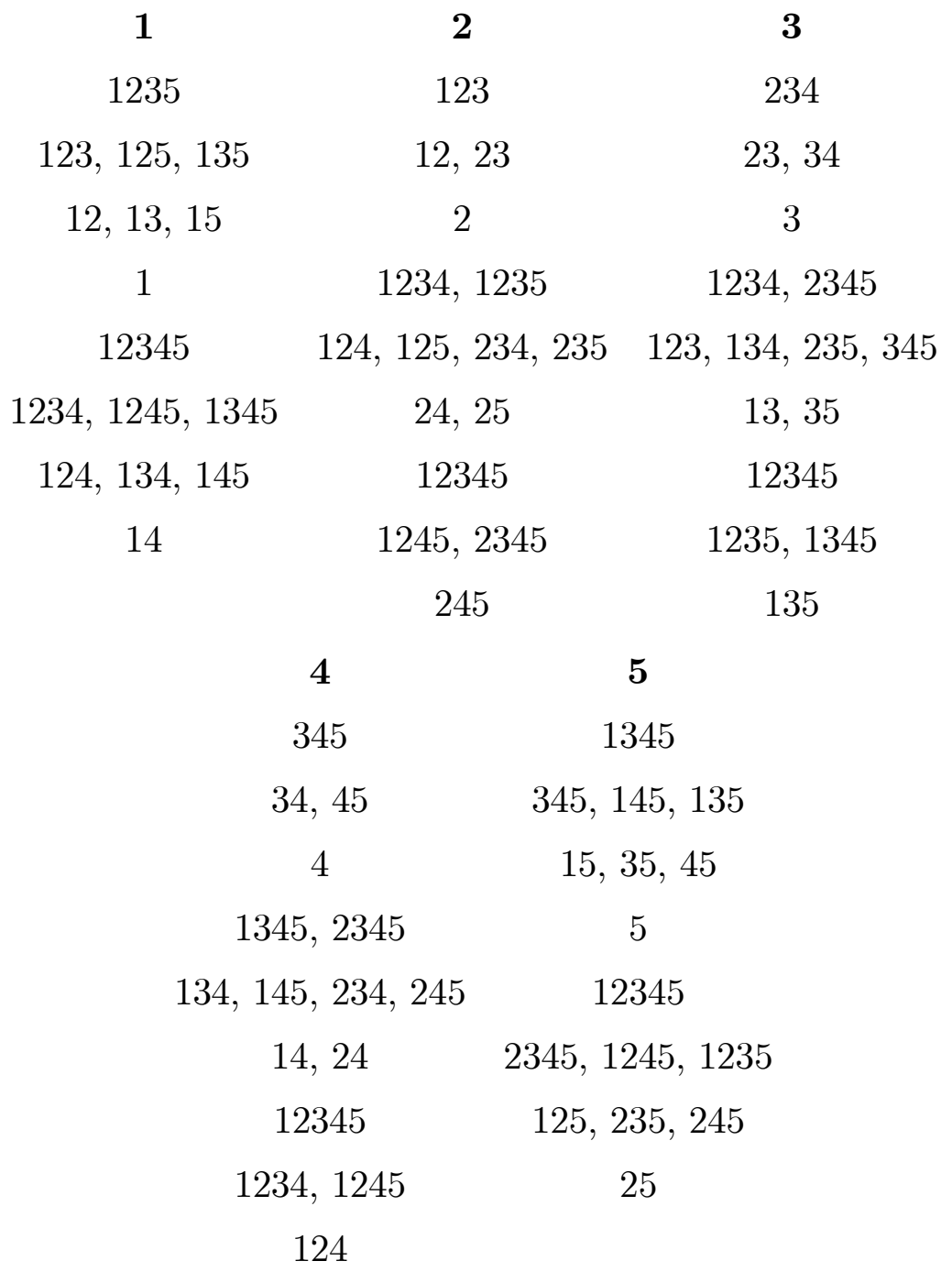

We first consider the weak top coalition property.

Definition 7 (Banerjee, Konishi, and Sönmez (2001)) Given a hedonic game 
$(N, P)$ and a player set $V \subseteq N$, a coalition $S \subseteq V$ is a weak top coalition for $V$ if $S$ has an ordered partition $\left\{S^{1}, \ldots, S^{\ell}\right\}$ such that

(1) $S \succeq_{i} T$ for all $i \in S^{1}$ and all $T \subseteq V$ with $i \in T$, and

(2) $T \succ_{i} S \Rightarrow T \cap\left(\bigcup_{m<k} S^{m}\right) \neq \emptyset$ for all $k>1$, all $i \in S^{k}$, and all $T \subseteq V$ with $i \in T$.

A hedonic game satisfies the weak top coalition property if every player set has a weak top coalition.

In order to see that the game in Example 3 does not satisfy this property, let's consider the player set $V=\{1,2,3\}$. Notice that no one of the singletons can be a weak top coalition for $V$ because it is not a top coalition for $V$ (i.e. it does not satisfy (1) in Definition 7). The same reason rules out all partitions of candidates for a weak top coalition that have a singleton at the first place. Because two of the players in $V$ (1 and 2) prefer $\{1,2,3\}$ to every doubleton consisting of players of $V$, all partitions of candidates for a weak top coalition that have a doubleton at the first place are ruled out as well. The whole set $V$ can not be a weak top coalition for itself because, let's say, $\{3\} \succ_{3}\{1,2,3\}$. Hence, $V$ has no weak top coalition, i.e. the game does not satisfy the weak top coalition property.

As we will see the same game does not satisfy the ordinal balancedness condition either.

Definition 8 (Scarf (1967), Bogomolnaia and Jackson (2002)) A family $\mathcal{B}$ of coalitions is called balanced if there exists a vector of positive weights $d_{C}$, such that for each player $i \in N, \sum_{C \in \mathcal{B}: i \in C} d_{C}=1$. A hedonic game $(N, P)$ is ordinally balanced if for each balanced family of coalitions $\mathcal{B}$ there exists a partition of $N$ such that for each $i \in N$ there exists a coalition $C \in \mathcal{B}$, $C \in \mathcal{N}_{i}$ such that $\mathcal{C}(i) \succeq_{i} C$. 
In other words, if we would like to check whether a game is ordinally balanced we have to find a partition of $N$ to each balanced family of coalitions, such that every player in that partition is weakly better off in comparison to her worst situation in the corresponding balanced family.

For the game in Example 3, let us take the following balanced family with a balanced weight $1 / 2$ for each coalition: $\mathcal{B}=\{\{1,2\},\{2,3\},\{3,4\},\{4,5\},\{1,5\}\}$. Notice that, given $\mathcal{B}$, all players do not like to remain single in a partition. Observe further that player 2 can be better off in a partition (in comparison to her worst situation in $\mathcal{B}$ ) if and only if that partition contains one of the coalitions $\{1,2\},\{2,3\},\{1,2,3\}$. Hence, the possible candidates for a partition are: $\{\{1,2\},\{3,4,5\}\}$, which is not liked by player 3 ; or $\{\{2,3\},\{1,4,5\}\}$, which is not liked by player 1 ; or $\{\{1,2,3\},\{4,5\}\}$, which is not liked again by player 3 . Hence, for the balanced family of coalitions $\mathcal{B}$ there is no suitable partition of $N$, i.e. the game is not ordinally balanced.

\section{References}

[1] Ballester, C. (2003): NP-completeness in hedonic games, Mimeo, Universitat Autònoma de Barcelona.

[2] Banerjee, S., H. Konishi, and T. Sönmez (2001): Core in a simple coalition formation game, Social Choice and Welfare 18, 135-153.

[3] Bogomolnaia, A. and M. O. Jackson (2002): The stability of hedonic coalition structures, Games and Economic Behavior 38, 201-230. 
[4] Burani, N. and W. S. Zwicker (2003): Coalition formation games with separable preferences, Mathematical Social Sciences 45, 27-52.

[5] Cechlárová, K. and J. Hajduková (2002): Computational complexity of stable partitions with B-preferences, International Journal of Game Theory $31,353-364$.

[6] Cechlárová, K. and A. Romero-Medina (2001): Stability in coalition formation games, International Journal of Game Theory 29, 487-494.

[7] Dréze, J. and J. Greenberg (1980): Hedonic coalitions: optimality and stability, Econometrica 48, 987-1003.

[8] Scarf, H. (1967): The core of an N-person game, Econometrica 35, 50-69.

[9] Tarjan, R. E. (1972): Depth-first search and linear graph algorithms, SIAM Journal of Computing 1(2), 146-160. 